\title{
A COVERING CONDITION FOR PRIME IDEALS
}

\author{
W. W. SMITH
}

Abstract. Commutative rings are studied which have the property that every ideal contained in the union of a collection of prime ideals is contained in one of the prime ideals of the collection. This property is shown to be equivalent to the condition that every prime ideal is the radical of a principal ideal.

All rings considered in this note will be commutative. Reis and Viswanathan [1] considered the following property:

( $\left.^{*}\right)$ if an ideal $I \subseteq \mathrm{U}_{\alpha \in S} P_{\alpha}$ where the $P_{\alpha}(\alpha \in S)$ are prime ideals of $R$ then $I \subseteq P_{\alpha}$ for some $\alpha \in S$.

The principal result of [1] is that a Noetherian ring satisfies $\left({ }^{*}\right)$ if and only if every prime ideal is the radical of a principal ideal. The theorem below shows the hypothesis that the ring be Noetherian is not needed.

Also in [1] the authors consider the "apparently weaker" condition:

(**) if a prime ideal $P \subseteq \bigcup_{\alpha \in S} P_{\alpha}$ when the $P_{\alpha}(\alpha \in S)$ are prime ideals of $R$ then $P \subseteq P_{\alpha}$ for some $\alpha \in S$.

The observation that for the $P_{\alpha}$ prime, the complement of $\bigcup_{\alpha \in S} P_{\alpha}$ is a multiplicative semigroup leads one to conclude if $I$ is any ideal contained in $\bigcup_{\alpha \in S} P$ then there is a prime ideal $P$ such that $I \subseteq P$ $\subseteq \bigcup_{\alpha \in S} P_{\alpha}$. It then follows that condition $\left({ }^{*}\right)$ and $\left({ }^{* *}\right)$ are equivalent.

TheOREM. A ring $R$ satisfies $\left(^{*}\right)$ if and only if every prime ideal of $R$ is the radical of a principal ideal in $R$.

Proof. We first show if $P$ is prime and $P$ is not the radical of a principal ideal then $P$ is contained in the union of a collection of prime ideals, none of which contain $P$. For each $r$ in $P$ since $P \neq \sqrt{ } r$ and since $\sqrt{ } r$ is the intersection of all of the prime ideals of $R$ which contain $r$, there is a prime $P_{r}$ such that $r \in P_{r}$ but $P \nsubseteq P_{r}$. Clearly $P \subseteq U_{r \in P} P_{r}$. Therefore, if $R$ satisfies $\left({ }^{*}\right)$ then every prime must be the radical of a principal ideal. Conversely, if $P \subseteq \mathrm{U}_{\alpha \in S} P_{\alpha}$ and $P=\sqrt{ } r$

Received by the editors February 25, 1971.

A MS 1970 subject classifications. Primary 13A15, 13F05; Secondary 13E05, 13F10, $13 \mathrm{~A} 10$.

Key words and phrases. Commutative ring, Noetherian ring, prime ideal. 
for some $r$ we must have $r$ in $\bigcup_{\alpha \in S} P_{\alpha}$. Therefore $r \in P_{\alpha}$ for some $\alpha$ in $S$ and hence $P=\sqrt{ } r \subseteq P_{\alpha}$. Thus we conclude $R$ satisfies condition $\left({ }^{* *}\right)$ and hence $\left(^{*}\right)$.

\section{REFERENCES}

1. Clive M. Reis and T. M. Viswanathan, A compactness property for prime ideals in Noetherian rings, Proc. Amer. Math. Soc. 25(1970), 353-356. MR 40 \#7244.

University of North Carolina, Chapel Hill, North Carolina 27514 